Niniejsza publikacja jest dostęna na licencji Creative Commons. Uznanie autorstwa-Użycie niekomercyjne-Bez utworów zależnych 3.0 Polska. Pewne prawa zastrzeżone na rzecz autora. Zezwala się na wykorzystanie publikacji zgodnie z licencja - pod warunkiem zachowania niniejszej informacji licencyjnej oraz wskazania autora jako właściciela praw do tekstu. Treść licencji jest dostępna na stronie: http://creativecommons.org/licenses/by-nc-nd/3.0/pl/

Lingwistyka Stosowana 18: 3/2016, 67-79

\author{
Artur Dariusz KUBACKI
}

Uniwersytet Pedagogiczny im. Komisji Edukacji Narodowej w Krakowie

\title{
Der plurizentrische Ansatz in der Rechtsübersetzung. Eine Fallstudie zur schweizerhochdeutschen und bundesdeutschen Terminologie im Familienrecht ${ }^{1}$
}

\begin{abstract}
:
A Pluricentric Approach to Legal Translation: the Case of Swiss German and German Family Law Terminology

The awareness of differences between Swiss German and German legal terminology is very important for legal translators. Unfortunately, terminological differences between standard German and its Swiss variety have not been sufficiently documented yet and many German-Polish dictionaries do not inform about them at all. On the other hand, there are dictionaries of Helvetisms on the market, but they rarely contain specialist vocabulary. Furthermore, Polish translators do not have easy access to legal texts in the Swiss German language, which restricts their possibilities of examining its specific character and consequently of finding out the differences in such linguistic areas as morphology, syntax, lexis or pragmatics. The basic reason for linguistic differences is that Germany and multilingual Switzerland are two different national organisms with separate political, economic, cultural and social systems. As a result, it is not possible to use standardized terminology because its differentiation serves to render dissimilar realities. This is why translators must be aware of the fact that while working with texts written in the Swiss German language they will come across not only terminological differences. It is enough to look at Swiss and German divorce decrees and statutory provisions in the area of family law to see the scale of difficulties translators are faced with. Hence prospective translators of German legal texts coming from various German-speaking countries should be sensitized in the course of their training to the phenomenon of pluricentrism of the German language.
\end{abstract}

\section{Einleitung}

Nach Meinung von E. Lang (2010: 197) rückt das Thema der Plurizentrik ${ }^{2}$ der deutschen Sprache seit den 1990er Jahren zunehmend ins allgemeine Bewusstsein. Die Konzeptualisierung des Deutschen als plurizentrischer Sprache umfasst nach R. de

${ }^{1}$ Der folgende Aufsatz stellt eine erweiterte Fassung meiner Publikation unter dem Titel Plurizentrik in der Fachübersetzung am Beispiel der schweizerischen Rechtssprache dar, herausgegeben von Jianhua Zhu, Jin Zhao und Michael Szurawitzki in den Akten des XIII. Internationalen Germanistenkongresses Schanghai 2015. Germanistik zwischen Tradition und Innovation, Publikationen der Internationalen Vereinigung für Germanistik (IVG), Peter Lang, Frankfurt a. M. etc. 2016, in Druck.

${ }^{2}$ Diese Erscheinung wird auch als Plurizentrismus oder Plurizentrizität genannt. 
Cillia (2006: 365) mindestens drei gleichwertige Sprachvarietäten: das bundesdeutsche Deutsch (auch Binnendeutsch), das österreichische Standarddeutsch (Österreichisch) und das Schweizerdeutsch ${ }^{3}$ (auch Schweizerhochdeutsch, Schweizer Standarddeutsch). In der Fachsprachenforschung und Translationswissenschaft ist der plurizentrische Ansatz jedoch bisher kaum reflektiert worden. Nach U. Ammon (1995: 515) gehören die Fachsprachen in der Nationalvarietätenforschung zu den wichtigen Forschungsdesideraten, die aus Raumgründen in seinem Buch unberücksichtigt bleiben mussten.

Bislang entstanden m. W. mehrere Arbeiten zur österreichischen Rechtssprache und deren Kodifizierung. In erster Linie sind hier das Wörterbuch der österreichischen Rechts-, Wirtschafts- und Verwaltungsterminologie von Heidemarie Markhardt (2006) sowie das Wörterbuch rechtsterminologischer Unterschiede Österreich-Deutschland von Rudolf Muhr und Marlene Peinhopf (2015) zu nennen. Wichtig für das Sprachpaar Deutsch-Polnisch ist zudem ein Verzeichnis häufiger Rechtsbegriffe mit ihren polnischen Entsprechungen, geordnet nach sechzehn Rechtsgebieten, herausgegeben vom Österreichischen Verband der Gerichtsdolmetscher (1997) unter dem Titel Die österreichische Rechtsterminologie (vgl. A.D. Kubacki 2015b: 21). Auch andere Fachsprachen, z.B. die Küchensprache (vgl. H.D. Pohl 2007), wurden in Österreich bereits gut kodifiziert.

Eine ähnlich umfangreiche Literatur, insbesondere Fachwörterbücher, fehlen jedoch für die schweizerischen Fachsprachen. Eine Ausnahme stellt nur die Publikation Schweizerisches juristisches Wörterbuch von Peter Metzger (zwei Auflagen 1996 und 2005) dar.

Beim Schweizerdeutschen sind primär die standardsprachliche Ebene und vereinzelt auch Fachtermini lexikalisiert worden. Entstanden sind so mehrere Schweizer Wörterbücher, z.B. die von Ingrid Bigler (1987), Kurt Meyer (2006), Hans Bickel und Christoph Landolt (2012).

Im Kontext des plurizentrischen Ansatzes darf natürlich auch das Variantenwörterbuch des Deutschen (U. Ammon et al. 2004) nicht vergessen werden. Es handelt sich dabei um einen völlig neuen Wörterbuchtyp, der auf dem Konzept einer plurizentrischen deutschen Standardsprache beruht. In diesem Wörterbuch, an dem namhafte Linguisten aus Deutschland, Österreich und der Schweiz mitgearbeitet haben, wurden möglichst alle nationalen und regionalen Varianten der deutschen Standardsprache und ihre gemeindeutschen Entsprechungen erfasst und in ihrer geographischen Verteilung dokumentiert. Es enthält ca. 12.000 Einträge mit spezifischen und unspezifischen Besonderheiten der Standardsprache in Österreich, der Schweiz und Deutschland, aber auch mit spezifischen Besonderheiten des Deutschen in Liechtenstein, Luxemburg, Südtirol und Ostbelgien. Allerdings bleiben fachsprachliche Ausdrücke wie auch veraltetes und dialektales Wortgut dort unberücksichtigt (U. Ammon et al. 2004: XI).

\footnotetext{
${ }^{3}$ Nach Auffassung von S. Firyn (2012: 52) soll das Schweizerdeutsch (Schwyzerdütsch) nicht mit dem Schweizerhochdeutsch (Schweizer Schriftdeutsch) verwechselt werden, weil das Schweizerdeutsch eine auf alemannischen Dialekten basierende Umgangssprache darstellt.
} 
Der schweizerischen Fachsprache wurde lediglich - außer dem Wörterbuch von Peter Metzger - in der Darstellung der Worthelvetismen von Ulrich Ammon (1995: 260-277) Rechnung getragen. Auf seinen Wortlisten sind nur wenige zentrale Themenbereiche wie (1) Speisen, Mahlzeiten, (2) Haushalt, Kleidung, (3) Verwaltung, Justiz, Gesundheitswesen, Schule, Militär, (4) Geschäftsleben, Handwerk, Landwirtschaft, Verkehr, (5) Sport, Spiele, (6) menschliches Verhalten, Soziales, Charaktereigenschaften, Körperteile und (7) Sonstiges vermerkt. Die Liste mit der schweizerischen Rechtssprache (3) ist jedoch sehr bescheiden und verzeichnet lediglich 103 Wörter und Wendungen.

Im folgenden Beitrag möchte ich versuchen, am Beispiel der schweizerischen und deutschen Rechtssprache aufzuzeigen, dass dem plurizentrischen Ansatz in der Fachübersetzung mehr Beachtung geschenkt werden sollte. Zuerst wird der Stand der wissenschaftlichen Diskussion über das Deutsche als plurizentrischer Sprache und die Gemengelage in der Deutschschweiz kurz geschildert. Im Anschluss daran wird die Plurizentrik am Beispiel von Scheidungsurteilen besprochen. Dabei werden die terminologischen Parallelen und Unterschiede zwischen dem schweizerischen und deutschen Familienrecht mit ihren polnischen Entsprechungen aufgezeigt. Auch die Makrostruktur der Scheidungsurteile aus der Schweiz und der Bundesrepublik Deutschland wird unter die Lupe genommen. Abschließend werden Schlussfolgerungen gezogen und Aussichten für die translatorische Praxis aufgezeigt.

\section{Deutsch als plurizentrische Sprache}

Bis in die 1980er Jahre des 20. Jahrhunderts galt Deutsch als monozentrische Sprache. Angenommen wurde, dass die deutsche Sprache nur ein Zentrum besitze und das in der Bundesrepublik Deutschland gesprochene Deutsch als Hauptvariante bzw. Norm anzusehen sei. Die Ausprägungen der deutschen Sprache in Österreich, der Schweiz, Luxemburg und Südtirol galten hingegen als Abweichung von der Norm („Randdeutsch“) (vgl. H. Moser 1985: 1678ff.). M. Kucharski (2009: 6) führt in seiner Diplomarbeit aus, dass man sich der monozentrischen Auffassung der deutschen Sprache Schritt für Schritt widersetzte und diese Entwicklung durch eine Reihe von Publikationen namhafter Germanisten gefördert wurde. Ohne Zweifel gehören dazu u.a. die Abhandlungen von H. Kloss (1978), M.G. Clyne (1984, 1995), U. Ammon (1991, 1995, 1998), R. Muhr (1991, 1997), W. Pollak (1994), A. Szulc (1999) und R. de Cillia (2006). Heute wird die deutsche Sprache in der germanistischen Linguistik als plurizentrische Sprache angesehen, d.h. als eine Sprache, die über mehrere Zentren verfügt. Amtssprache ist sie in Deutschland, Österreich, der Schweiz und Liechtenstein, als regionale Amtssprache tritt sie in mehreren angrenzenden Regionen wie Ostbelgien, Luxemburg und Südtirol auf (vgl. K. Meyer 2006: 15). Allerdings gibt es bis heute keine einheitliche Definition des Terminus Plurizentrik.

W. Pollak (1994: 12) assoziiert die Plurizentrik mit dem Qualifikationsmerkmal der hochdeutschen Standardsprache. Er erklärt das wie folgt: „Der Terminus 'plurizentrisch' [...] stellt gleichsam die 'Magna Charta' für die Anerkennung der drei staatsnationalen Varietäten des Deutschen in Österreich, in der BRD und der 
deutschsprachigen Schweiz (Austriazismen, Teutonismen, Helvetismen) dar und impliziert deren absolute Gleichberechtigung. [...].“

Nach Ansicht von M.G. Clyne (1995: 20), einem in der Nationalvarietätenforschung bekannten Germanisten aus Australien, gehört Deutsch wie Englisch, Französisch und andere Sprachen auch zu den plurizentrischen Sprachen, die auf mehrere, einander beeinflussende Zentren verteilt sind. Jedes Zentrum hat seine eigene nationale Varietät (national variety) mit zumindest einigen kodifizierten Normen. Zentren entstehen auf der Basis von Sprechergruppen und/ oder Sprachgebieten. Eine besondere Form der Zentren sind Nationen. Dann spricht man von Plurinationalität, die nach U. Ammon (1998: 319) nur ein Teil der Plurizentrik einer Sprache ist. Darüber hinaus ist nach M.G. Clyne (1995: 21) die Erscheinung der Asymmetrie plurizentrischer Sprachen auf den soziolinguistischen Status der einzelnen Varietäten zurückzuführen. Es handelt sich dabei einerseits um die Einstellungen der Sprecher zur Standardsprache, andererseits um historische, politische und wirtschaftliche Machtverhältnisse zwischen den genannten Sprachzentren (vgl. Ch. Messina 2015: 61f.). In diesem Kontext unterscheidet M.G. Clyne (1995: 21f.) im Falle der deutschen Sprache zwischen den jeweils dominanten Nationen (DNationen) wie Deutschland und den anderen Nationen (A-Nationen) wie Österreich. So werden einige Varianten sowohl in Bayern als auch in Österreich verwendet, wobei sie in Deutschland als regional und in Österreich als national standardsprachlich angesehen werden.

Bei der Beschreibung plurizentrischer Sprachen erörtert U. Ammon (1991: 17f.) auch den Begriff „Zentrum“. Er definiert sie als Sprachen, die mindestens zwei Standardvarietäten in verschiedenen Zentren besitzen, wobei sich die Zentren auf mindestens zwei Nationen verteilen. Für U. Ammon (1996: 158) besitzt eine Sprache dann ein nationales Zentrum, wenn dieses eine eigene Standardvarietät hat. Mit einer eigenen Standardvarietät eines Staates hat man es nur dann zu tun, wenn „sprachliche Besonderheiten der betreffenden Standardvarietät speziell für den betreffenden Staat geschaffen wurden." (idid.) Dazu komme es dann, wenn ein Sprachzentrum eine endonormative Rolle spiele. Mit anderen Worten handelt es sich um einen Staat, der eigene, amtlich verbindliche normative Rechtschreib-, Aussprache-, Grammatikregeln etc. ausgearbeitet hat, also über einen „linguistischen Kodex" verfügt (vgl. M. Kucharski 2009: 7f.).

U. Ammon (1995: 96) differenziert bei plurizentrischen Sprachen zwischen Voll- und Halbzentren einer Sprache. Beide verfügen über eigene nationale Varianten und Varietäten, erstere allerdings auch über einen Binnenkodex für ihre Standardvarietät (Vollzentrum $=$ endonormatives Zentrum), letztere hingegen nicht (Halbzentrum $=$ exonormatives Zentrum). Bei der Binnenkodifizierung der jeweiligen Varietät liegt ein Sprachkodex vor, der in dem jeweiligen Zentrum erarbeitet und verlegt, aber nicht zwangsläufig dort auch gedruckt wird. Für Österreich wären hier das Österreichische Wörterbuch, für Deutschland der Duden und für die deutschsprachige Schweiz Unser Wortschatz. Schweizer Wörterbuch der deutschen Sprache (I. Bigler 1987) oder Schweizer Wörterbuch. So sagen wir in der Schweiz (K. Meyer 2006) anzuführen (vgl. M. Kucharski 2009: 9). Die deutsche Sprache verfügt somit über drei Vollzentren: Deutschland, Österreich und die deutschspra- 
chige Schweiz. Halbzentren sind: Liechtenstein, Luxemburg, die deutschsprachige Region in Ostbelgien und Südtirol. Grundsätzlich sind nach U. Ammon (1995: 95) auch transnationale Zentren möglich. Als Beispiel sei hier von ihm das sog. EUDeutsch genannt, weil es auch seine eigenen Besonderheiten herausgebildet und in eigenen Wörterbüchern wie dem IATE lexikalisiert hat.

U. Ammon (1995: 61ff.) hat zu dem Themenkomplex „Sprachzentrum“ eine umfassende Terminologie entwickelt, die auch auf andere plurizentrische Sprachen angewendet werden kann. Die grundlegenden Begriffe sind: Varietät, Standardvarietät, Variante, Variable, Sprachkodex, Standardsprache, Nichtstandardsprache, Gebrauchsstandard. Aus Platzgründen kann hier nur auf seine wissenschaftlichen Beiträge verwiesen werden.

In der Fachliteratur zum Thema Plurizentrik lassen sich nach U. Ammon (1998: 313ff.) drei Deutungsmuster unterscheiden, die von M. Kucharski (2009: 8f.) folgendermaßen charakterisiert werden:

1. Plurizentrisch-nationales (plurizentrisches) Modell geht von der absoluten Gleichberechtigung der drei staatsnationalen Varietäten des Deutschen in Österreich, in der BRD und der deutschsprachigen Schweiz aus. Die verschiedenen Zentren der deutschen Sprache werden hier mit verschiedenen Nationen gleichgesetzt. Vertreter dieser Lesart sind M.G. Clyne, R. Muhr, R. Wodak, W. Pollak und R. de Cillia.

2. Plurizentrisch-integrales (plurinationales) Modell geht davon aus, dass der überwiegende Teil der deutschen Schrift- und Standardsprache in Deutschland, Österreich und der deutschsprachigen Schweiz übereinstimmt und dass diese Staaten bzw. Landesteile über einen beschränkten Variantenbestand verfügen, der aber die einzelnen Varietäten der deutschen Sprache bildet. Im Gegensatz zum plurizentrisch-nationalen Modell werden die verschiedenen Zentren der deutschen Sprache nicht mit den verschiedenen Nationen gleichgesetzt, sondern es wird zwischen „staatlichem Zentrum“ und „,nationalem Zentrum“ als Unterbegriffen zum Oberbegriff „Zentrum“ unterschieden (vgl. U. Ammon 1998) [...]. Vertreter dieser Leseart sind P. Wiesinger, J. Ebner, P. Polenz und U. Ammon.

3. Pluriareales Modell betrifft nur die österreichische Varietät. Es erkennt österreichische Merkmale des Deutschen an. Mit Ausnahme der Verwaltungsterminologie, die nur innerhalb des Staatsgebietes gilt, wird von den Vertretern dieses Modells das österreichische Deutsch nicht als ,national einheitliche Sprachform“, sondern als „eine durch die Eigenstaatlichkeit Österreichs bedingte süddeutsche Variante" des Deutschen verstanden [...]. Vertreter dieser Leseart sind N. Wolf, H. Scheuringer und H.D. Pohl.

Beim zweiten Modell überschreitet die Nation die Staatsgrenzen, beim dritten Modell die Sprache. Abschließend ist noch auf die Bezeichnung der Besonderheiten der nationalen Varianten hinzuweisen. Nach Ansicht von T. Wissik (2014: 15) werden für die nationalen Varianten Österreichs und der Schweiz unproblematische Termini angewendet wie Austriazismen und Helvetismen. Für die sprachlichen Besonderheiten Deutschlands werden in den anderen Zentren teils negativ konnotierte, daher nicht unproblematische Benennungen wie Teutonismen, Deutschlandismen 
und Germanismen (Germanizismen) verwendet. Die Teutonismen werden von T. Wissik bevorzugt, weil sie unter Linguisten auf mehr Akzeptanz stoßen.

\section{Die sprachliche Gemengelage in der Deutschschweiz}

Die in der Bundesverfassung verankerten Landessprachen der Schweizerischen Eidgenossenschaft sind Deutsch, Französisch, Italienisch und Rätoromanisch. T. Wissik (2014: 33f.) gibt an, dass rund 63\% der Gesamtbevölkerung respektive $72 \%$ der Schweizer Bevölkerung deutschsprachig sind, was ca. 4,6 Millionen deutschsprachigen Einwohnern entspricht. Von den 26 Kantonen haben siebzehn Kantone Deutsch als alleinige Amtssprache, vier mehrsprachige Kantone gebrauchen Deutsch neben einer (oder mehreren) anderen Sprache(n) als Amtssprache.

Das in der Schweiz gesprochene Deutsch, das Schweizerhochdeutsch, gilt heute als nationale Varietät (vgl. U. Ammon 1995: 5). Die für die Schweiz typischen Besonderheiten der deutschen Sprache, welche in den Großwörterbüchern des Deutschen und in eigenen Spezialwörterbüchern beschrieben sind, werden als Helvetismen bezeichnet (vgl. K. Meyer 2006: 16). Nach B. Siebenhaar und A. Wyler (1997: 9) gibt es in der Deutschschweiz zwei Formen des Deutschen: die Standardsprache (auch Schrift- oder Hochdeutsch genannt) und die schweizerische Mundart, das sog. Schwyzertütsch, gegliedert in zahlreiche, regional verschiedene Dialekte. Erstere wird vor allem geschrieben und gelesen, letztere gesprochen und nur selten verschriftlicht. B. Siebenhaar und A. Wyler (1997: 9f.) bezeichnen diese Sprachsituation in der Schweiz als Diglossie und stellen fest:

In einer Sprachgemeinschaft werden zwei Formen der gleichen Sprache verwendet, eine hochsprachliche und eine volkssprachliche, und jede Sprachform hat unterschiedliche Geltungsbereiche. Dabei sind die Sprachformen immer deutlich voneinander unterschieden, Misch- und Übergangsformen gibt es kaum. Deutschschweizern ist immer bewusst, welche Sprachform sie verwenden. Wenn sie in bestimmten Situationen zur Hochsprache wechseln (wechseln müssen), so wird das auch meist thematisiert. Ein allmähliches Hinübergehen vom Dialekt in die Hochsprache gibt es nicht. (ibid.)

Es gibt demnach keine nationale Umgangssprache, wie sie in den deutschen und österreichischen Varianten gebräuchlich ist (vgl. K. Meyer 2006: 19). Viele Deutschschweizer empfinden die Mundart als etwas Schweizerisches, etwas Eigenes, die Standardsprache als etwas Fremdes und haben eine gewisse Scheu, die Standardsprache zu verwenden (vgl. T. Wissik 2014: 39f.). Abschließend ist nach K. Meyer (2006: 16f.) zu unterstreichen, dass das Schweizerdeutsche aus plurizentrischer Sicht kein minderes oder unreines Standarddeutsch, sondern die schweizerische Standardvarietät des Deutschen ist. Ihre nationalen und regionalen Besonderheiten sind als gleichberechtigte standardsprachliche Erscheinungsformen des Deutschen einzustufen.

\section{Plurizentrik in der Rechtsübersetzung}

In der germanistischen Literatur untersucht man die Merkmale des Schweizerdeutschen auf den Ebenen der Lautung, Schreibung, Flexion, Wortbildung, Syntax, Pragmatik, Semantik und Lexik (vgl. K. Meyer 2006: 19, A.D. Kubacki 2012: 179). 
Für die Zwecke des vorliegenden Beitrags sind hauptsächlich die Ebenen Lexik und Pragmatik von Bedeutung.

Als Basis für den übersetzungsrelevanten Vergleich dienen die in der deutschen und schweizerischen Rechtspflege verwendeten Terminologien. Sie finden in deutschen und schweizerischen Gerichtsurteilen Anwendung. Das untersuchte Korpus umfasst jeweils zehn deutsche und schweizerische Urteile bzw. Entscheide zu Ehescheidungen und anderen Ehesachen aus verschiedenen Regionen Deutschlands und der deutschsprachigen Schweiz.

Die gesetzlichen Regelungen im Familienrecht, denen übersetzungsrelevante Lexik entnommen wird, sind im deutschen Bürgerlichen Gesetzbuch (BGB) und teilweise in der deutschen Zivilprozessordnung (ZPO) sowie im schweizerischen Zivilgesetzbuch (ZGB) enthalten. Als Paralleltext und somit eine sehr gute Quelle für terminologische Recherchen dient dem Fachübersetzer das polnische Familienund Vormundschaftsgesetzbuch.

Nach H. Bickel und Ch. Landolt (2012: 83) besitzt jedes Staatswesen unzählige eigenständige Institutionen, eine eigene Rechtsprechung und im Falle der deutschsprachigen Länder jeweils lange selbständige Verwaltungstraditionen.

Bezüglich der deutschen Rechtssprache zählt G.-R. de Groot (1999: 204) folgende Varietäten auf: eine österreichisch-deutsche, eine deutsch-deutsche, eine schweizerisch-deutsche, eine liechtensteinisch-deutsche, eine belgisch-deutsche, eine italienisch-deutsche und eine EU-deutsche. Letztere hat über Verordnungen und Direktiven direkten Einfluss auf die Sprachgestaltung der nationalen Rechtssysteme.

Ein terminologischer Vergleich des Familienrechts ist nicht nur eine sprachliche, sondern zugleich eine komparatistische Aufgabe. Nach P. Sandrini (1996: 145ff.) kann eine Äquivalenz der Begrifflichkeiten verschiedener Rechtssysteme nur partiell oder approximativ erreicht werden.

Die Übersetzung schweizerischer Urteile aus dem Deutschen ins Polnische wird durch die sehr schwache Kodifizierung der Helvetismen in bilingualen Fachwörterbüchern erschwert. Nach meinen jüngsten Untersuchungen (vgl. A.D. Kubacki 2015a) sind lediglich von 14 bis zu 45 Lexeme pro 268 untersuchte Helvetismen, also von $5 \%$ bis zu $17 \%$ der Helvetismen, je nach Wörterbuch kodifiziert.

Viele Rechtsinstitute im Familienrecht existieren sowohl in der Schweiz als auch in Deutschland, und auch im polnischen Recht finden sich dafür Entsprechungen. Das erklärt sich wohl durch das römische Recht als gemeinsame Grundlage des Gesetzgebungsverfahrens im jeweiligen Staat. Daher können solche Termini mühelos ins Polnische übertragen werden wie: Scheidung (rozwód), Lebensgemeinschaft (wspólne pożycie), Ehevertrag (małżeńska umowa majątkowa), Vormund (opiekun), elterliche Sorge/Sorgerecht (władza rodzicielska) oder Gütertrennung (rozdzielność majątkowa) (vgl. A.D. Kubacki 2014: 173).

Einige Rechtsinstitute bestehen in Deutschland und in der Schweiz, werden aber unterschiedlich bezeichnet, z.B. ${ }^{4} \mathrm{CH}$ : Scheidungsbegehren (pozew o rozwód) - D: Scheidungsantrag (wniosek o rozwód). Das hängt vom jeweiligen Verfahren und der

\footnotetext{
${ }^{4}$ Die folgenden Abkürzungen bezeichnen die entsprechenden Länder: $\mathrm{CH}=$ Schweiz, $\mathrm{D}=$ Deutschland und PL $=$ Polen.
} 
hierfür entwickelten Terminologie ab: In der Schweiz ist es ein streitiges Verfahren und in Deutschland ein nichtstreitiges Verfahren. Dies betrifft auch die abweichende Benennung von Institutionen in der Rechtspflege, z.B.: CH: Vormundschaftsbehörde (sąd opiekuńczy) - D: Vormundschaftsgericht (sąd opiekuńczy).

Auf der Basis einer Analyse deutscher und schweizerischer Gerichtsentscheidungen sowie familienrechtlicher Gesetzestexte können viele terminologische Unterschiede nachgewiesen werden. In den Gesetzestexten (Tabelle 1) und in den Gerichtsurteilen (Tabelle 2) finden sich folgende Lexeme (hier mit ihren polnischen Übersetzungsvorschlägen):

\begin{tabular}{|c|c|c|}
\hline Schweiz & Deutschland & Polen \\
\hline Scheidungsbegehren & Scheidungsantrag & $\begin{array}{l}\text { pozew o rozwód } \\
\text { (Scheidungsklage) }\end{array}$ \\
\hline $\begin{array}{l}\text { Scheidung auf } \\
\text { gemeinsames Begehren }\end{array}$ & $\begin{array}{l}\text { einverständliche Schei- } \\
\text { dung }\end{array}$ & $\begin{array}{l}\text { rozwód za porozumieniem } \\
\text { stron (Scheidung mit Einver- } \\
\text { ständnis der Parteien) }\end{array}$ \\
\hline elterliche Sorge & $\begin{array}{l}\text { (elterliche) Sorge, } \\
\text { elterliches Sorgerecht }\end{array}$ & $\begin{array}{l}\text { władza rodzicielska } \\
\text { (wörtlich: elterliche Macht) }\end{array}$ \\
\hline $\begin{array}{l}\text { tiefe und unheilbare Zerrüt- } \\
\text { tung der Ehe }\end{array}$ & Scheitern der Ehe & $\begin{array}{l}\text { zupełny i trwały rozkład poży- } \\
\text { cia małżeńskiego (völlige und } \\
\text { dauerhafte Ehezerrüttung) }\end{array}$ \\
\hline Hausrat & $\begin{array}{l}\text { Gegenstände des } \\
\text { ehelichen Haushalts }\end{array}$ & $\begin{array}{l}\text { przedmioty zwykłego urząd- } \\
\text { zenia domowego (Gegenstän- } \\
\text { de der gewöhn-lichen Haus- } \\
\text { einrichtung) }\end{array}$ \\
\hline Vormundschaftsbehörde & $\begin{array}{l}\text { Familiengericht, } \\
\text { früher: Vormundschafts- } \\
\text { gericht }\end{array}$ & $\begin{array}{l}\text { sąd opiekuńczy } \\
\text { (Vormundschaftsgericht) }\end{array}$ \\
\hline Kind & (Adoptiv)kind & $\begin{array}{l}\text { dziecko przysposobione } \\
\text { (adoptiertes/angenommenes } \\
\text { Kind) }\end{array}$ \\
\hline Adoption & $\begin{array}{l}\text { Annahme an } \\
\text { Statt, Adoption }\end{array}$ & adopcja (Adoption) \\
\hline ordentlicher Güterstand & $\begin{array}{l}\text { Güterstand der } \\
\text { Zugewinngemeinschaft }\end{array}$ & $\begin{array}{l}\text { ustawowy ustrój majątkowy } \\
\text { (gesetzlicher Güterstand) }\end{array}$ \\
\hline Eigengut & Anfangsvermögen & $\begin{array}{l}\text { majątek osobisty } \\
\text { (persönliches Vermögen) }\end{array}$ \\
\hline Errungenschaft & Endvermögen & $\begin{array}{l}\text { majątek wspólny } \\
\text { (gemeinsames Vermögen) }\end{array}$ \\
\hline
\end{tabular}

Tabelle 1: Beispiele für die terminologischen Unterschiede in den Gesetzestexten 


\begin{tabular}{|l|l|l|}
\hline \multicolumn{1}{|c|}{ Schweiz } & \multicolumn{1}{|c|}{ Deutschland } & \multicolumn{1}{c|}{ Polen } \\
\hline $\begin{array}{l}\text { Alimente, } \\
\text { Unterhaltsbeitrag }\end{array}$ & $\begin{array}{l}\text { Unterhalt, } \\
\text { Unterhaltszahlung }\end{array}$ & $\begin{array}{l}\text { alimenty, } \\
\text { świadczenie alimentacyjne } \\
\text { (Unterhaltsleistung) }\end{array}$ \\
\hline Gerichtsschreiber & Protokollführer & $\begin{array}{l}\text { protokolant sądowy } \\
\text { (Gerichtsprotokollant) }\end{array}$ \\
\hline Einschreibegebühr & Gerichtskostenvorschuss & $\begin{array}{l}\text { wpis tymczasowy } \\
\text { (Übergangsgebühr) }\end{array}$ \\
\hline Rechtskraftbescheinigung & Rechtskraftzeugnis & $\begin{array}{l}\text { klauzula prawomocności } \\
\text { (Rechtskraftklausel) }\end{array}$ \\
\hline persönliche Effekten & private bewegliche Habe & $\begin{array}{l}\text { rzeczy osobiste } \\
\text { (persönliche Sachen) }\end{array}$ \\
\hline Aussöhnungsversuch & Versöhnungsversuch & $\begin{array}{l}\text { próba pojednania } \\
\text { (Versöhnungsversuch) }\end{array}$ \\
\hline Eheregister & $\begin{array}{l}\text { Familienbuch } \\
\text { ab 1.01.2009: Ehebuch }\end{array}$ & $\begin{array}{l}\text { księga małżenstw } \\
\text { (Ehebuch) }\end{array}$ \\
\hline Scheidungskonvention & Scheidungsvereinbarung & $\begin{array}{l}\text { umowa rozwodowa } \\
\text { (Scheidungsvertrag) }\end{array}$ \\
\hline Zivilstandsamt & Standesamt & $\begin{array}{l}\text { urząd stanu cywilnego } \\
\text { (Zivilstandsamt) }\end{array}$ \\
\hline Zivilstandsbeamter & Standesbeamter & $\begin{array}{l}\text { urzędnik stanu cywilnego } \\
\text { (Zivilstandsbeamter) }\end{array}$ \\
\hline Advokaturbüro & Rechtsanwaltskanzlei & $\begin{array}{l}\text { kancelaria adwokacka } \\
\text { (Rechtsanwaltskanzlei) }\end{array}$ \\
\hline
\end{tabular}

Tabelle 2: Beispiele für die terminologischen Unterschiede in den Gerichtsurteilen

Aus der vorgenannten Analyse geht hervor, dass alle terminologischen Unterschiede nach ihrer Benennungsmotivik wie folgt gruppiert werden können (vgl. A.D. Kubacki 2014: 166f.):

1. Gerichtsentscheidungen: $\mathrm{CH}$ : Entscheid-D: Beschluss (postanowienie),

2. Gerichtsverfahren: CH: Verfahren auf einseitiges Vorbringen - D: Angelegenheiten der freiwilligen Gerichtsbarkeit (postępowanie procesowe),

3. Parteien/Verfahrensbeteiligte: CH: Kläger/Beklagter (powód/pozwany) - D: Antragsteller/Antragsgegner (wnioskodawca/uczestnik postępowania),

4. Organe der Gerichtsbarkeit: D: Amtsgericht - CH: (je nach Kanton) Amts/Bezirks-/Kreis-/Regional-/Kantonalgericht (sąd pierwszej instancji),

5. Mitarbeiter des Gerichts: CH: Gerichtsschreiber - D: Justizangestellter als Urkundsbeamter der Geschäftsstelle (sekretarz sądowy),

6. Bestandteile einer Gerichtsentscheidung: CH: Erwägungen - D: Tatbestand (stan faktyczny).

Auch lexikalische Differenzen spiegeln sich durch Anwendung anderer formelhafter Wendungen in der Rechtssprache wider, was besonders in deutschen und schweizerischen Gerichtsentscheidungen $\mathrm{zu}$ sehen ist, z.B. in Gerichtsurteilen, Rechtsbelehrungen und Kostenentscheidungen. Formelhafte Wendungen in der Rechtsprache bezeichnen nach M. Kucharski (2009: 40) ,immer wiederkehrende Phrasen bzw. Wortverbindungen, die auf eine gewisse Gleichförmigkeit und Stereo- 
typizität des Verfahrens, auf das sie sich beziehen, hinweisen.“ Auch für T. Wissik (2014: 87) sind sie ein charakteristisches Merkmal der Rechts- und Verwaltungssprache. Als Beispiel der formelhaften Wendung führt sie die oft verwendete Phrase unter Bezugnahme auf an. Die formelhafte Rechtssprache dient im Falle bestimmter Rechtsinstitute der Verfahrensökonomie, Vereinheitlichung und Vergleichbarkeit von Verfahrensabläufen. Nach S. Pommer (2006: 26) haben auch Sprachmittler bei der Übertragung derartiger formelhafter Wendungen keine Formulierungsfreiheit, sondern sollten die entsprechenden Formulierungen, soweit in der Zielsprache vorhanden, verwenden. Dies wird durch folgende drei Beispiele aus dem Korpus belegt: 1.

D: $\quad$ Die Kosten des Verfahrens werden gegeneinander aufgehoben.

$\mathrm{CH}: \quad$ Allfällige Parteikosten werden wettgeschlagen.

PL: $\quad$ Koszty postępowania/wszelkie koszty stron znosza się wzajemnie.

2.

D: $\quad$ Vorstehendes Urteil ist rechtskräftig seit dem ...

CH: Vorstehendes Urteil ist am ... in Rechtskraft erwachsen.

PL: Powyższy wyrok jest prawomocny z dniem ...

3.

D: $\quad$ Ein Versorgungsausgleich findet nicht statt.

PL: Nie dojdzie do wyrównania zabezpieczenia roszczeń emerytalno-rentowych.

$\mathrm{CH}: \quad$ Zufolge Ehevertrags mit Gütertrennung (gemäss der im Ehevertrag vereinbarten Gütertrennung) erklären sich die Parteien güterrechtlich als auseinandergesetzt.

PL: $\quad$ Strony oświadczaja, że zgodnie z ustalona $w$ majatkowej umowie matżeńskiej rozdzielnościa majątkowa rozchodza się bez jakichkolwiek roszczeń wobec siebie wynikajacych z matzeńskiego prawa majątkowego.

Die Stereotypizität der Gerichtssprache fällt besonders bei der vom Gericht vorgenommenen Kostenentscheidung auf, die in fast allen Gerichtsentscheidungen enthalten ist (Beispiel Nr. 1). Auch die Bestätigung der Rechtskraft (Beispiel Nr. 2) ob formeller oder materieller Art - wird in den Rechtssystemen verglichener Staaten mittels feststehender Wendungen ausgedrückt. Formelhaften Charakter weisen auch die ehelichen/nachehelichen Vermögensfragen in den Gerichtsurteilen auf (Beispiel Nr. 3).

Die Plurizentrik macht sich auch in der Makrostruktur der schweizerischen, deutschen und polnischen Scheidungsurteile bemerkbar. Die differente Rechtswirklichkeit determiniert auch die unterschiedlichen Bestandteile im Rubrum des Urteils im jeweiligen Staat. Die Begründung eines Urteils ist kein obligatorischer Teil in der Schweiz und in Polen, aber fester Bestandteil jedes deutschen Scheidungsurteils. $\mathrm{Zu}$ erwähnen ist auch die Tatsache, dass Urteile in Deutschland im Namen des Volkes und in Polen im Namen der Republik Polen ergehen. In der Schweiz gibt es keine derartige Formulierung. Nur das Schweizerische Bundesgericht spricht seine Urteile im Namen der jeweiligen Abteilung, in der die Urteile gefällt werden. Die Differenzen betreffen auch die graphische Struktur eines Urteils (Angaben zu Aktenzeichen, Parteien und ihren Bevollmächtigten, Tag und Ort der Ausfertigung des Urteils, 
Rechtskraftbescheinigung usw., Anwendung von Aufzählungen in polnischen Urteilen, Zwischentiteln in deutschen und schweizerische Urteilen).

Ebenfalls auf der pragmatischen Ebene lassen sich die Unterschiede in der Verwendung von akademischen Graden und Titeln in der geschriebenen Sprache feststellen. In Deutschland und der Schweiz werden sie in den Gerichtsurteilen vergleichsweise weniger häufig als in Polen oder Österreich eingesetzt (vgl. T. Wissik 2014: 18 und 36).

\section{Schlussfolgerungen und Aussichten}

Die Ergebnisse lassen sich in folgenden sechs Punkten zusammenfassen:

1. U. Ammons Modelle zur Beschreibung von Helvetismen sind im Bereich der Rechtssprache nur bedingt anwendbar.

2. Es gibt nicht nur terminologische Unterschiede, sondern auch Gemeinsamkeiten und Überschneidungen der deutschen, schweizerischen und polnischen Rechtssprache.

3. Im Falle von Rechtstermini kann in den meisten Fällen nur von einer funktionalen, nicht aber von einer begrifflichen Äquivalenz gesprochen werden.

4. Bei der Übersetzung formelhafter Rechtssprache haben die Sprachmittler keine Formulierungsfreiheit und sollten sich der analogen Formulierungen bedienen, soweit diese in der Zielsprache vorhanden sind.

5. Auch die Makrostruktur von Gerichtsentscheidungen darf beim Übersetzen nicht außer Acht gelassen werden.

6. Wünschenswert wäre die Erstellung eines zweisprachigen Rechtswörterbuchs, in dem schweizerische und deutsche Rechtstermini geklärt wie auch substanzielle Unterschiede zwischen den beiden Rechtssystemen hervorgehoben werden.

Außerdem wäre es sehr interessant zu eruieren, inwiefern plurizentrische Sprachen translatorisches Handeln bzw. translatorische Entscheidungen beeinflussen und ob sie auch in der translatorischen Praxis eines Fachübersetzers relevant sind.

\section{Bibliographie}

Ammon, U. (1991), Die Plurizentrizität der deutschen Sprache. In: B. Ekmann/ H. Häuser/ P. Forsch/ W. Wucherpfennig (Hrsg.): Deutsch - eine Sprache? Wie viele Kulturen? Kopenhagen etc., 14-34.

Ammon, U. (1995), Die deutsche Sprache in Deutschland, Österreich und der Schweiz. Berlin etc.

Ammon, U. (1998), Plurinationalität oder Pluriarealität? Begriffliche und terminologische Präzisierungsvorschläge zur Plurizentrizität des Deutschen - mit einem Ausblick auf ein Wörterbuchprojekt. In: P. Ernst/ E. Patocka (Hrsg.), Deutsche Sprache in Raum und Zeit, Festschrift für Peter Wiesinger zum 60. Geburtstag. Wien, 313-322. 
Ammon, U. et al. (Hrsg.) (2004), Variantenwörterbuch des Deutschen. Die Standardsprache in Österreich, der Schweiz und Deutschland sowie in Liechtenstein, Luxemburg, Ostbelgien und Südtirol. Berlin.

Bickel, H./ Ch. Landolt (2012), Schweizerhochdeutsch. Wörterbuch der Standardsprache in der deutschen Schweiz. Berlin etc.

Bigler, I. (1987), Unser Wortschatz. Schweizer Wörterbuch der deutschen Sprache. Mit einem umfassenden Textteil zu Wortgebrauch und Grammatik. Zürich.

Clyne, M.G. (1984), Language and Society in the German-speaking countries. Cambridge etc.

Clyne, M.G. (1992), German as a Pluricentric Language. In: M. G. Clyne (Hrsg.), Pluricentric Languages. Different Norms in Different Countries. Berlin etc., $117-147$.

Clyne, M.G. (1995), The German Language in a Changing Europe. Cambridge.

de Cillia, R. (2006), Varietätenreiches Deutsch. Deutsch als plurizentrische Sprache und DaF-Unterricht. In: H.-J. Krumm/ P. Portmann-Tselikas (Hrsg.), Begegnungssprache Deutsch - Motivation, Herausforderung, Perspektiven. Innsbruck etc., $51-65$.

de Groot, G.-R. (1999), Zweisprachige juristische Wörterbücher. In: P. Sandrini (Hrsg.), Übersetzen von Rechtstexten. Fachkommunikation im Spannungsfeld zwischen Rechtsordnung und Sprache. Tübingen, 203-227.

Die österreichische Rechtsterminologie. Verzeichnis wichtiger Rechtsbegriffe geordnet nach Rechtsgebieten Deutsch-Polnisch. 1997, Österreichischer Verband der Gerichtsdolmetscher Wien.

Firyn, S. (2012), Regionalizmy w przekładzie na podstawie ttumaczeń niemieckopolskich. In: Rocznik Przekładoznawczy 7, 51-64.

Kloss, H. (1978), Die Entwicklung neuer germanischer Kultursprachen seit 1800. Düsseldorf.

Kubacki, A.D. (2012), Ttumaczenie poświadczone. Status, kształcenie, warsztat $i$ odpowiedzialność tłumacza przysięgłego. Warszawa.

Kubacki, A.D. (2014), Pluricentryzm w niemieckim jezyku standardowym i specjalistycznym. In: Comparative Legilinguistics. International Journal for Legal Communication 17, 163-182.

Kubacki, A.D. (2015a), Pluricentryzm w niemiecko-polskich słownikach ogólnych $i$ specjalistycznych. In: Lingwistyka Stosowana/ Applied Linguistics/ Angewandte Linguistik 15, 33-46.

Kubacki, A.D. (2015b), Wybór dokumentów austriackich dla kandydatów na ttumaczy przysięgtych. Chrzanów.

Kubacki, A.D. (2016), Plurizentrik in der Fachübersetzung am Beispiel der schweizerischen Rechtssprache. In J. Zhu/ J. Zhao/ M. Szurawitzki (Hrsg.), Akten des XIII. Internationalen Germanistenkongresses Schanghai 2015. Germanistik zwischen Tradition und Innovation. Frankfurt a. M. etc., in Druck.

Kucharski, M. (2009), Austriazismen im Erb- und Familienrecht. Diplomarbeit. Eingereicht am Zentrum für Translationswissenschaft der Universität Wien.

Lang, E. (2010), Plurizentrik als Übersetzungsproblem. In: I. Bartoszewicz/ M. Dalmas/ J. Szczęk/ A. Tworek (Hrsg.), Germanistische Linguistik extra 
muros - Aufforderungen. Linguistische Treffen in Wrocław; 5. Wrocław/ Dresden, 197-203.

Markhardt, H. (2006), Wörterbuch der österreichischen Rechts-, Wirtschafts- und Verwaltungsterminologie. Frankfurt a. M. etc.

Messina, Ch. (2015), Die österreichischen Wirtschaftssprachen. Terminologie und diatopische Variation. Berlin.

Metzger, P. (1996), Schweizerisches juristisches Wörterbuch einschliesslich Versicherungsrecht mit Synonymen und Antonymen. Basel etc.

Metzger, P. (2005), Schweizerisches juristisches Wörterbuch einschliesslich Versicherungsrecht mit Synonymen und Antonymen. Basel etc.

Meyer, K. (2006), Schweizer Wörterbuch. So sagen wir in der Schweiz. Mit einem Beitrag von Hans Bickel. Frauenfeld etc.

Moser, H. (1985), Die Entwicklung der deutschen Sprache seit 1945. In: W. Besch/ O. Reichmann/ S. Sonderegger (Hrsg.), Sprachgeschichte. Ein Handbuch zur Geschichte der deutschen Sprache und ihrer Forschung. Berlin, 1678-1707.

Muhr, R. (1991), Sprachnormen in Österreich und anderswo. Zur sprachlichen Identität der Österreicher und zur Bestimmung von Standardsprachen in plurizentrischen Sprachen. In: Znanstvena Revija 1,3. Akten des jugoslawischösterreichischen Germanistentreffens im Rahmen der Alpen Adria vom 11.13.11.1989 an der Pädagogischen Fakultät der Universität Maribor. Maribor, 73-77.

Muhr, R. (1997), Zur Terminologie und Methode der Beschreibung plurizentrischer Sprachen und deren Varietät am Beispiel des Deutschen. In: R. Muhr/ R. Schrodt (Hrsg.), Österreichisches Deutsch und andere nationale Varietäten plurizentrischer Sprachen in Europa. Empirische Analysen. Wien, 40-67.

Muhr, R./ M. Peinhof (2015), Wörterbuch rechtsterminologischer Unterschiede Österreich-Deutschland. Frankfurt a. M. etc.

Pohl, H.D. (2007), Die österreichische Küchensprache. Ein Lexikon der typisch österreichischen kulinarischen Besonderheiten (mit sprachwissenschaftlichen Erläuterungen). Wien.

Pollak, W. (1994), Österreich und Europa: sprachkulturelle und nationale Identität. Wien.

Pommer, S. (2006), Rechtsübersetzung und Rechtsvergleichung. Translatologische Fragen zur Interdisziplinarität. Frankfurt a. M. etc.

Sandrini, P. (1996), Terminologiearbeit im Recht. Deskriptiver begriffsorientierter Ansatz vom Standpunkt des Übersetzers. Wien.

Siebenhaar, B./ A. Wyler (1997), Dialekt und Hochsprache in der deutschsprachigen Schweiz. Zürich.

Szulc, A. (1999), Odmiany narodowe języka niemieckiego. Geneza - rozwój-perspektywy. Kraków.

Wissik, T. (2014), Terminologische Variation in der Rechts- und Verwaltungssprache. Deutschland - Österreich - Schweiz. Berlin. 